\title{
A Method of the Best Approximation by Fractal Function
}

\author{
Yong-Suk Kang, Myong-Gil Rim \\ Faculty of Mathematics, Kim Il Sung University, Pyongyang, DPR Korea
}

Email address:

Ryongnam19@yahoo.com (Yong-Suk K.)

\section{To cite this article:}

Yong-Suk Kang, Myong-Gil Rim. A Method of the Best Approximation by Fractal Function. International Journal of Theoretical and Applied Mathematics. Vol. 3, No. 1, 2017, pp. 11-18. doi: 10.11648/j.ijtam.20170301.12

Received: September 7, 2016; Accepted: November 5, 2016; Published: December 9, 2016

\begin{abstract}
We present a method constructing a function which is the best approximation for given data and satisfiesthe given self-similar condition. For this, we construct a space F of local self-similar fractal functions and show its properties. Next we present a computational scheme constructing the best fractal approximation in this space and estimate an error of the constructed fractal approximation. Our best fractal approximation is a fixed point of some fractal interpolation function.
\end{abstract}

Keywords: Fractal Interpolation, Fractal Approximation, Iterated Function System, Fractal Function Space

\section{Introduction}

Fractal approximation has been applied to model the objects which have fractal characteristics in nature. Fractal functions whose graphs are fractal sets have been widely used in approximation theory, signal processing, interpolation theory,computer graphics and so on. Hence, constructions of fractal functions and fractal approximation have been studied in many papers.

Constructions of fractal functions by fractal interpolation have been introduced by many researchers. A construction of one variable fractal interpolation functions by the iterated function system (IFS) with a data set on R was studied in [1, $2,14]$, where the constructed fractal functions were self-similar ones. The construction was generalized in [3, 4, 17], which constructed local self-similar fractal functions. Constructions of bivariate fractal interpolation functions (BFIFs) have been studied in [5-11, 13, 17]. A construction of BFIFs by fractal interpolation on $\mathrm{R}$ was presented in $[5,17]$ and self-affine fractal interpolation functions were constructed by IFS with a data set on a triangular domain in [12]. Constructions of self-similar BFIFs in $[9,11]$ and self-affine BFIFs in $[10,13]$ by IFS with a data set on a rectangular grid were introduced. In [6], local self-similarBFIFs were constructed by the recurrent iterated function system(RIFS) on a rectangular grid. A construction of local self-similar fractal interpolation functions in $\mathrm{R}^{\mathrm{n}}$ was studied in [4].

To construct fractal interpolation we need a data set $\left\{\left(x_{i}, y_{i}\right), i=0,1, \ldots, n\right\}$ and a set of scale parameters $\left\{s_{i}, i=1, \ldots, n\right\}$. The fractal property of the graph of the interpolation function is determinated by those data. Let a division of the interval and scale parameters be given, that is, a fractal property of the function be given. If the number of experimental data is more than the number of the interval division, then we can not construct the fractal interpolation for the data using fractal interpolation theory.

So we assume that a division of the interval and scale parameters be given (that is, a fractal property of the function) and study the problem constructing the best fractal approximation for the data set $\left\{\left(\bar{x}_{i}, \bar{z}_{i}\right), i=0,1, \ldots, m\right\}$, where $m>n$ ( $n$ is the number of the interval division).

In $[15,18]$, constructionsofthebest approximation of functions by the fractal functions were presented, respectively. Butthe continuity of the approximation was not guaranteed then. The best fractal approximation of a continuous function in $L^{2}$ space was introduced in [16]. In [7], a space of differentiable fractal interpolation functions was constructed and it was proved that the constructed space is the reproducing kernel Hilbert space.

We construct a space of fractal interpolation functions with a given division of the interval and scale parameters and find a function satisfying some approximation condition for data $\left\{\left(\bar{x}_{i}, z_{i}\right), i=0,1, \ldots, m\right\}$, with $m>n$ in this space. We call it a local self-similar fractal approximation. The values of the function at nodes of division $\left\{y_{i}, i=0,1, \ldots, n\right\}$ are unknown unlike interpolation function.

The rest of the article is organized as follows: Section 2 constructs a linear space $\mathcal{F}$ of local self-similar fractal functions and then a linear space $\mathcal{T}$ of contraction 
operators which is isomorphic to the constructed $\mathcal{F}$. Section 3 proves that there exists a unique contraction operator (RB operator) in $\mathcal{T}$ corresponding to LSFAin $\mathcal{F}$. We change the problem constructing this RB operator to the problem constructing $\left\{y_{i}, i=0,1, \ldots, n\right\}$ and the fixed point of this operator is LSFA in $\mathcal{F}$ of a given data set. Section 4 estimates an error of the constructed fractal approximation and Section 5 gives examples of calculation of the least squares fractalapproximation of a coastline.

\section{A Space of Local Self-Similar Fractal Functions and a Space of Contractive Operators}

In this section, we construct a space of local self-similar fractal functions and a space of contraction operators which are isomorphic to each other.

Let

$$
\begin{gathered}
\Delta=\left\{x_{i} \in \mathrm{R}: i=0,1, \cdots, n\right\}, \\
a=x_{0}<x_{1}<\ldots<x_{n}=b, \\
I=[a, b], \quad I_{i}=\left[x_{i-1}, x_{i}\right], \\
\left\{s_{i}:\left|s_{i}\right|<1, i=1,2, \cdots, n\right\}
\end{gathered}
$$

be given.

Let $1<q \leq n(q \in \mathrm{N})$,

$$
J_{k}=\left[x_{s(k)}, x_{e(k)}\right], x_{s(k)}, x_{e(k)} \in\left\{x_{0}, x_{1}, \cdots, x_{n}\right\}
$$

And $e(k)-s(k) \geq 2, \quad k=1, \cdots, q . I_{i}$ is called a region and $J_{k}$ a domain. We define a mapping $\gamma:\{1, \ldots, n\} \rightarrow\{1, \ldots, q\}$, which means that we relate every region to a domain. For each $i \in\{1, \ldots, n\}$, denote $k=\gamma(i)$.

For $i \in\{1, \ldots, n\}$, define a mapping $u_{i, k}: J_{k} \rightarrow I_{i}$ by

$$
u_{i, k}(x)=a_{i} x+b_{i}
$$

which satisfies

$$
u_{i, k}\left(x_{s(k)}\right)=x_{i-1}, u_{i, k}\left(x_{e(k)}\right)=x_{i} .
$$

Let $f(\in C(I))$ be a continuous function satisfying

$$
f(x)=s_{i} \cdot f\left(u_{i, k}^{-1}(x)\right)+p_{i, k}\left(u_{i, k}^{-1}(x)\right), x \in I_{i}
$$

where functions $p_{i, k}: J_{k} \rightarrow I_{i}, \quad i=1, \ldots, n$ are defined by $p_{i, k}(x)=c_{i} x+d_{i}$ and satisfy the following conditions:

$$
\begin{gathered}
s_{i} f\left(x_{s(k)}\right)+p_{i, k}\left(x_{s(k)}\right)=f\left(x_{i-1}\right) \\
s_{i} f\left(x_{e(k)}\right)+p_{i, k}\left(x_{e(k)}\right)=f\left(x_{i}\right)
\end{gathered}
$$

Define a space of functions satisfying the equations (4), (5) by $\mathcal{F}$. The graph of $f \in \mathcal{F}$ has a local self-similarity and we get $f(x) \equiv 0 \in \mathcal{F}$ which corresponds to $c_{i}=0, d_{i}=0$, $i \in\{1, \ldots, n\}$.

Lemma $1 \mathcal{F}$ is a linear subspace of dimension $n+1$ of $C(I)$.

Proof. For $f, \tilde{f} \in \mathcal{F}$ and $\lambda \in R$, we have

$$
\begin{gathered}
f(x)=s_{i} \cdot f\left(u_{i, k}^{-1}(x)\right)+p_{i, k}\left(u_{i, k}^{-1}(x)\right), \\
\tilde{f}(x)=s_{i} \cdot \widetilde{f}\left(u_{i, k}^{-1}(x)\right)+\widetilde{p}_{i, k}\left(u_{i, k}^{-1}(x)\right), \quad x \in I_{i} .
\end{gathered}
$$

Hence

$$
\begin{aligned}
(f+\tilde{f})(x) & :=s_{i} \cdot(f+\tilde{f})\left(u_{i, k}^{-1}(x)\right)+\left(p_{i, k}+\tilde{p}_{i, k}\right)\left(u_{i, k}^{-1}(x)\right), \quad x \in I_{i}, \\
(\lambda f)(x) & :=s_{i} \cdot(\lambda f)\left(u_{i, k}^{-1}(x)\right)+\left(\lambda p_{i, k}\right)\left(u_{i, k}^{-1}(x)\right), \quad x \in I_{i} .
\end{aligned}
$$

Thus $f+\widetilde{f} \in \mathcal{F}$ and $\lambda f \in \mathcal{F}$.

Because for $f \in \mathcal{F}, \quad\left(f\left(x_{0}\right), f\left(x_{1}\right), \ldots, f\left(x_{n}\right)\right) \in R^{n+1}$ is uniquely determined, a mapping $\Psi: \mathcal{F} \rightarrow R^{n+1}$ is defined by

$$
\Psi(f)=\left(f\left(x_{0}\right), f\left(x_{1}\right), \ldots, f\left(x_{n}\right)\right) .
$$

And for $\left(y_{0}, y_{1}, \ldots, y_{n}\right) \in R^{n+1}$, there exists a unique $f \in \mathcal{F}$ such that $f\left(x_{i}\right)=y_{i}, i=1, \ldots, n$.

In fact, the existence and uniquenssof $f$ areensured by the existence and uniquenss of the recurrent fractal interpolation function ([3]).

This shows that the mapping $\Psi: \mathcal{F} \rightarrow R^{n+1}$ is a bijection. We can easily check that the mapping $\Psi$ is linear. Hence $\mathcal{F}$ and $R^{n+1}$ are isomorphic. A basis of $\mathcal{F}$ is

$$
\Psi^{-1}\left(e_{i}\right), e_{i}=(0, \ldots, 1,0, \ldots, 0), i=1, \ldots, n+1 .
$$

The space $\mathcal{F}$ is a Banach space with the norm $\|\cdot\|_{\infty}$.

For a $y=\left(y_{0}, y_{1}, \ldots, y_{n}\right) \in R^{n+1}$, define a function space $\mathcal{F}_{y}$ by

$$
\mathcal{F}_{y}=\left\{f \in C(I): f\left(x_{i}\right)=y_{i}, \quad i=1, \ldots, n\right\} .
$$

Then $\left(\mathcal{F}_{y},\|\cdot\|_{\infty}\right)$ is a complete space.

For $f \in \mathcal{F}_{y}$, define a function $T_{y} f: I \rightarrow R$ by

$$
\left(T_{y} f\right)(x):=s_{i} \cdot f\left(u_{i, k}^{-1}(x)\right)+p_{i, k}^{T_{y}}\left(u_{i, k}^{-1}(x)\right), \quad x \in I_{i},
$$

where $\quad p_{i, k}^{T_{y}}(x)=c_{i, k}^{T_{y}} x+d_{i, k}^{T_{y}} \quad$ satisfies $\quad$ the following conditions:

$$
\left(T_{y} f\right)\left(x_{i-1}\right)=y_{i-1},\left(T_{y} f\right)\left(x_{i}\right)=y_{i}
$$


i.e.

$$
s_{i} y_{s(k)}+p_{i, k}^{T_{y}}\left(x_{s(k)}\right)=y_{i-1}, s_{i} y_{e(k)}+p_{i, k}^{T_{y}}\left(x_{e(k)}\right)=y_{i} .
$$

By the seconditions, $c_{i, k}^{T_{y}}$ and $d_{i, k}^{T_{y}}$ are uniquely givenby

$$
\begin{gathered}
c_{i, k}^{T_{y}}=\frac{\left(y_{i}-y_{i-1}\right)-s_{i}\left(y_{e(k)}-y_{s(k)}\right)}{x_{e(k)}-x_{s(k)}}, \\
d_{i, k}^{T_{y}}=\frac{y_{i-1} x_{e(k)}-y_{i} x_{s(k)}+s_{i}\left(x_{s(k)} y_{e(k)}-y_{s(k)} x_{e(k)}\right)}{x_{e(k)}-x_{s(k)}} .
\end{gathered}
$$

Therefore, for a $y=\left(y_{0}, y_{1}, \ldots, y_{n}\right) \in R^{n+1}$, we get a unique operator $T_{\mathbf{y}}$. And because $T_{\mathrm{y}} f$ is continuous by (12), $\quad T_{y} f \in \mathcal{F}_{y}$. Thustheoperator $\quad T_{y}: \mathcal{F}_{y} \rightarrow \mathcal{F}_{y} \quad$ is defined by (12). It is easy to verify that the operator $T_{y}$ isacontraction with respect to $\|\cdot\|_{\infty}$. According to the fixed-point theorem in a complete space, there exists a unique $f_{T_{y}} \in \mathcal{F}_{y}$ such that

$$
\left(f_{T_{y}}\left(x_{0}\right), f_{T_{y}}\left(x_{1}\right), \cdots, f_{T_{y}}\left(x_{n}\right)\right)=\left(y_{0}, y_{1}, \cdots, y_{n}\right)
$$

Let $\mathcal{T}$ betheset of such operators. Define a mapping $\Phi: \mathbf{R}^{n+1} \rightarrow \mathcal{T}$ by $\Phi(y)=T_{y} \in \mathcal{T}$. Then the mapping $\Phi$ is a bijection.

Lemma 2. $\mathcal{T}$ is a linear space of dimensionn +1 .

Proof. For $\mathrm{T}_{\mathrm{y}_{1}}, \mathrm{~T}_{\mathrm{y}_{2}} \in \mathrm{T}$ and $\lambda \in R$, define $T_{\mathrm{y}_{1}}+T_{\mathbf{y}_{2}}$ on $\mathcal{F}_{y_{1}+y_{2}}$ by $f \in \mathcal{F}_{y_{1}+y_{2}}$,

$$
\left(T_{\mathbf{y}_{1}}+T_{\mathbf{y}_{2}}\right)(f)(x):=s_{i} \cdot f\left(u_{i}^{-1}(x)\right)+\left(p_{i}^{T_{y_{1}}}+p_{i}^{T_{\mathbf{y}_{2}}}\right)\left(u_{i}^{-1}(x)\right), \quad x \in I_{i}
$$

and $\lambda T_{\mathbf{y}_{1}}$ on $\mathcal{F}_{\lambda y_{1}}$ by

$$
\begin{array}{r}
\tilde{f} \in \mathcal{F}_{\lambda y_{1}}, \quad\left(\lambda T_{\mathbf{y}_{1}}\right)(\tilde{f})(x):=s_{i} \cdot \tilde{f}\left(u_{i}^{-1}(x)\right)+\lambda p_{i}^{T_{\mathbf{y}_{1}}}\left(u_{i}^{-1}(x)\right), \\
x \in I_{i}, \quad i=1, \quad \ldots, \quad n
\end{array}
$$

Note that we omit a subscript $k$ after this because the domain and region are all fixed.

It is clear that $\left(T_{y_{1}}+T_{y_{2}}\right) f \in \mathcal{F}_{y_{1}+y_{2}} \quad$ and $\left(\lambda T_{y_{1}}\right) \tilde{f} \in \mathcal{F}_{\lambda y_{1}}$. Therefore, $T_{y_{1}}+T_{y_{2}} \in \mathcal{T}$ and $\lambda T_{y_{1}} \in \mathcal{T}$, i.e. the linear operations are defined in the set $\mathcal{T}$. It is easy to prove that the set $\mathcal{T}$ is a linear space with respect to the linear operations.

The mapping $\Phi: R^{n+1} \rightarrow \mathcal{T}$ is linear. In fact, because for

$$
\begin{aligned}
& \mathbf{y}_{1}=\left(y_{1,0}, \quad y_{1,1}, \cdots, \quad y_{1, n}\right), \\
& \mathbf{y}_{2}=\left(y_{2,0}, \quad y_{2,1}, \cdots, \quad y_{2, n}\right) \in \mathbf{R}^{n+1}
\end{aligned}
$$

and $\lambda \in \mathrm{R}$, by (15), (16)

$$
\begin{array}{r}
p_{i}^{T_{\mathrm{y}_{1} \mathrm{y}_{2}}}(x)=c_{i}^{T_{\mathrm{y}_{1} \mathrm{y}_{2}}} x+d_{i}^{T_{\mathrm{y}_{1}+\mathrm{y}_{2}}}=\left(c_{i}^{T_{\mathrm{y}_{1}}}+c_{i}^{T_{\mathrm{y}_{2}}}\right) x+\left(d_{i}^{T_{\mathrm{y}_{1}}}+d_{i}^{T_{\mathrm{y}_{2}}}\right) \\
=c_{i}^{T_{\mathrm{Y}_{1}}} x+d_{i}^{T_{\mathrm{y}_{1}}}+c_{i}^{T_{\mathrm{y}_{2}}} x+d_{i}^{T_{\mathrm{y}_{2}}}=p_{i}^{T_{\mathrm{y}_{1}}}(x)+p_{i}^{T_{\mathrm{y}_{2}}}(x), \\
p_{i}^{T_{\lambda y_{1}}}(x)=c_{i}^{T_{\lambda y_{1}}} x+d_{i}^{T_{\lambda y_{1}}}=\lambda\left(c_{i}^{T_{\mathrm{y}_{1}}} x+d_{i}^{T_{\mathrm{y}_{1}}}\right)=\lambda p_{i}^{T_{\mathrm{y}_{1}}}(x)
\end{array}
$$

and we get

$$
\begin{gathered}
\left(\Phi\left(\mathbf{y}_{1}+\mathbf{y}_{2}\right)\right)(f)(x)=\left(\Phi\left(\mathbf{y}_{1}\right)+\Phi\left(\mathbf{y}_{2}\right)\right)(f)(x) \\
\left(\Phi\left(\lambda \mathbf{y}_{1}\right)\right)(f)(x)=\lambda\left(\Phi\left(\mathbf{y}_{1}\right)\right)(f)(x) .
\end{gathered}
$$

Hence, $\mathcal{T}$ and $\mathrm{R}^{n+1}$ are isomorphic, which means that the dimension of $\mathcal{T}$ is $\mathrm{n}+1$.

By the isomorphic relation, $(0,0, \cdots, 0) \in R^{n+1}$ correspondedstothe operator $T$ defined by

$$
(T f)(x)=s_{i} \cdot f\left(u_{i}^{-1}(x)\right), x \in I_{i},
$$

whose fixed point is $f_{T}(x) \equiv 0$.

Theorem 1. Let $\mathcal{F}$ and $\mathcal{T}$ be the linear spaces constructed above. Then they are isomorphic.

Proof. This follows from Lemmas 1 and 2.

Denote the isomorphism of $\mathcal{F}$ to $\mathcal{T}$ by $\widetilde{\Psi}$. Note that for $f \in \mathcal{F}$, the fixed point of $T$ with $\widetilde{\Psi}(f)=T$ is $f$.

\section{Construction of LSFA of a Data Set}

In this section, we prove that there exists the least squares fractal approximation $f$ in $\mathcal{F}$ of a data setand present an algorithm for finding $f$ by calculating approximately the contraction operatorTin $\mathcal{T}$ corresponding to $f$.

Let $P$ be a data set given by

$$
P=\left\{\left(\bar{x}_{i}, z_{i}\right): i=0,1, \ldots, m\right\},
$$

$$
\left(\bar{x}_{0}<\bar{x}_{1}<\cdots<\bar{x}_{m}, \bar{x}_{0}=x_{0}, \bar{x}_{m}=x_{n}\right)
$$

where $m>n$.

An $f^{*}$ is called the least squares fractal approximation (LSFA) if $f^{*}$ is a solution of the following question:

$$
\min _{f \in \mathcal{F}} \sum_{i=0}^{m}\left(f\left(\bar{x}_{i}\right)-\bar{z}_{i}\right)^{2}
$$

First, we consider theexistence and uniqeness of LSFA.

Theorem 2. If $\left\{x_{0}, x_{1}, \ldots, x_{n}\right\} \subset\left\{\bar{x}_{0}, \ldots, \bar{x}_{m}\right\}$, then there exist a unique solution $f^{*} \in \mathcal{F}$ of (26) and a unique $T_{f^{*}} \in \mathcal{T}$ whose fixed point is $f^{*}$.

Proof. Define an operator $B_{m}: \mathcal{F} \rightarrow R^{m+1}$ by

$$
f \in \mathcal{F}, \quad B_{m} f=\left(f\left(\bar{x}_{0}\right), f\left(\bar{x}_{1}\right), \cdots, f\left(\bar{x}_{m}\right)\right)
$$


And denote $B_{m} \mathcal{F}$ by $\mathfrak{D}$. Then $B_{m}$ is a linear operator and $\mathcal{D}$ is a linear subspace of $R^{m+1}$. The question (26) is represented by

$$
\min _{f \in \mathcal{F}}\left\|B_{m} f-\hat{\mathbf{z}}\right\|_{E}^{2}
$$

where $\hat{\mathbf{z}}=\left\{\bar{z}_{0}, \bar{z}_{1}, \ldots, \bar{z}_{m}\right\} \in R^{m+1}$ and $\|\cdot\|_{E}$ is the Euclidean norm.

Therefore, (26) is equivalent to the following question:

$$
\min _{z \in \mathcal{D}}\|\mathbf{z}-\hat{\mathbf{z}}\|_{E}^{2}
$$

Because $\left(R^{m+1},\|\cdot\|_{E}\right)$ is a Hilbert space and $\mathscr{D}$ is a subspace of $R^{m+1}$, there exists a unique solution $z^{*}$ of (29). If $B_{m} f \equiv 0$, then from the hypothesis of the theorem

$$
\left(f\left(x_{0}\right), f\left(x_{1}\right), \cdots, f\left(x_{n}\right)\right)=(0,0, \ldots, 0)
$$

And $f(x) \equiv 0$ by the construction of $\mathcal{F}$. Therefore, $B_{m}$ is an injection and there exists a unique $f^{*} \in \mathcal{F}$ such that $B_{m} f^{*}=z^{*}$, i.e. there exists a unique $T^{*}=\widetilde{\Psi}^{-1}\left(f^{*}\right) \in \mathcal{T}$.

From Theorem 2, Equation (26) is equivalent to the following question:

$$
\min _{T \in \mathcal{T}}\left\|B_{m} f_{T}-\hat{\mathbf{z}}\right\|_{E}^{2}
$$

Now, we consider a construction of the LSFA.

Let $\Psi$ be the linear mapping defined by (9) and denote

$v_{i}=\Psi^{-1}\left(\mathbf{e}_{i}\right), \mathbf{e}_{i}=(0, \ldots, 1,0, \ldots, 0), \quad i=1, \ldots, n+1$

Then $\left\{v_{i}\right\}_{i=1}^{n+1}$ is a basis of $\mathcal{F}$ and there exist unique $\lambda_{1}, \ldots, \lambda_{n} \in \mathrm{R}$ such that $f^{*}=\sum_{k=0}^{n} \lambda_{k} v_{k}$. For $f, g \in \mathcal{F}$, define $<f, \quad g>\in \mathrm{R}$ by $\langle f, g\rangle=\sum_{k=0}^{m} f\left(\bar{x}_{i}\right) \cdot g\left(\bar{x}_{i}\right)$.

We get a normal equation

$$
\begin{gathered}
A \alpha=b, \\
A=\left(a_{i j}\right), a_{i j}=<v_{i}, v_{j}>, b=\left(b_{i}\right), b_{i}=\sum_{k=0}^{m} z_{k} v_{i}\left(\bar{x}_{k}\right)
\end{gathered}
$$

to find $f^{*} \in \mathcal{F}$.

Since $v_{i}, i=1, \cdots, n$ are fractal functions in (34), it needs enormous operations. Therefore, we consider an algorithm for calculating the approximation of contractionoperator $T_{f^{*}}$. Wecalculateapproximately $f^{*}$ as the fixed point of $T_{f^{*}}$.
Now, for $p_{0}=\left(\bar{x}_{0}, \bar{x}_{1}, \ldots, \bar{x}_{m}\right)$, let us denote $X_{m}=p_{0} \times R^{m+1}$. Define an operator $T_{m}$ on $X_{m}$ by

$$
\begin{gathered}
z=\left(z_{0}, z_{1}, \ldots, z_{m}\right) \in R^{m+1}, T_{m}\left(p_{0}, z\right)=\left(p_{0}, \tilde{z}\right), \\
\widetilde{z}=\left(\widetilde{z}_{0}, \widetilde{z}_{1}, \ldots, \widetilde{z}_{m}\right),
\end{gathered}
$$

where $\widetilde{z}_{i}, i=1,2, \cdots, m$ are defined as follows:for $\bar{x}_{i}$, there exist $l(\in\{1, \cdots, n\})$ and $k(\in\{0,1, \ldots, m-1\})$ such that $\bar{x}_{i} \in I_{l}$ and $\bar{x}_{k} \leq u_{l}^{-1}\left(\bar{x}_{i}\right) \leq \bar{x}_{k+1}$. Then

$$
\widetilde{z}_{i}=s_{l}\left(z_{k}+z_{k+1}\right) / 2+c_{l} u_{l}^{-1}\left(\bar{x}_{i}\right)+d_{l} .
$$

The operator $T_{m}$ is given by $s_{i}, c_{i}$ and $d_{i}$, $i=1,2, \ldots, n$, where $\left|s_{i}\right|<1, i=1,2, \ldots, n$ and $c_{i}, d_{i}$, $i=1,2, \ldots, n$ are calculated by (15), (16) and represented by $y_{0}, y_{1}, \ldots, y_{n}$. Let us denote $\left(T_{m}\left(p_{0}, z\right)\right)_{2}=\widetilde{z}$.

We find a $T_{m}^{*}$ such that

$$
\left\|\left(T_{m}\left(p_{0}, \hat{z}\right)\right)_{2}-\hat{z}\right\|_{E}=\|\tilde{\hat{z}}-\hat{z}\|_{E} \rightarrow \min
$$

This problem is a minimization problem of a multi-variable function with unknown $y_{0}, y_{1}, \ldots, y_{n}$. We find $y_{0}, y_{1}, \ldots, y_{n}$ from this problem. Next we find the RB operator $T_{m}^{*}$ using the method constructingthe fractal interpolation and its fixed point, that is the fractal interpolation with $\left\{\left(x_{0}, y_{0}\right\},\left\{x_{1}, y_{1}\right\}, \ldots,\left\{x_{n}, y_{n}\right\}\right\}$ and scale parameters $s_{1}, s_{2}, \ldots, s_{n}$, is our best fractal approximation.

\section{Estimation for Errors of the Approximation}

In this section, we consider a relation between $T_{m}$ and $T$ and estimatean error betweenthe approximation solution $f_{T^{*}}$ and given data.

For $\left(p_{0}, y\right),\left(p_{0}, g\right) \in X_{m} \quad$ and $\quad \lambda \in R \quad, \quad$ define $\left(p_{0}, y\right)+\left(p_{0}, g\right), \lambda\left(p_{0}, y\right)$ and $\left\|\left(p_{0}, y\right)\right\|$ as follows:

$$
\begin{gathered}
\left(p_{0}, y\right)+\left(p_{0}, g\right):=\left(p_{0}, y+g\right), \lambda\left(p_{0}, y\right):=\left(p_{0}, \lambda y\right) \\
\left\|\left(p_{0}, y\right)\right\|:=\max _{0 \leq i \leq m}\left|y_{i}\right|
\end{gathered}
$$

Lemma 3. $T_{m}$ is a contraction operator on $X_{m}$. Proof. For

$$
\left(p_{0}, y\right),\left(p_{0}, g\right) \in X_{m}
$$

we get 


$$
\begin{gathered}
\left|\bar{y}_{i}-\bar{g}_{i}\right|=\frac{s_{l}}{2}\left|y_{k}+y_{k+1}-g_{k}-g_{k+1}\right|, \\
\left\|\mathrm{T}_{\mathrm{m}}\left(\mathrm{p}_{0}, \mathrm{y}\right)-\mathrm{T}_{\mathrm{m}}\left(\mathrm{p}_{0}, \mathrm{~g}\right)\right\|=\left\|\left(\mathrm{p}_{0}, \overline{\mathrm{y}}\right)-\left(\mathrm{p}_{0}, \overline{\mathrm{g}}\right)\right\| \\
=\left\|\left(\mathrm{p}_{0}, \overline{\mathrm{y}}-\overline{\mathrm{g}}\right)\right\|=\max _{\mathrm{i}}\left|\overline{\mathrm{y}}_{\mathrm{i}}-\overline{\mathrm{g}}_{\mathrm{i}}\right|, \\
\leq \frac{s_{l}}{2}\left(\left|y_{k}-g_{k}\right|+\left|y_{k+1}-g_{k+1}\right|\right) \\
\leq s_{i} \max _{i}\left|y_{i}-g_{i}\right|=s_{i}\left\|\left(p_{0}, y-g\right)\right\|,
\end{gathered}
$$

where $c=\max \left\{\left|s_{1}\right|, \ldots,\left|s_{n}\right|\right\}<1$. Therefore, $T_{m}$ is a contraction operator with contraction constant $c$.

Because $X_{m}$ is equivalent to $R^{m+1}$, we identify $X_{m}$ with $R^{m+1}$ and get a diagram that shows the relation between $T$ and $T_{m}$ (see Fig 1).

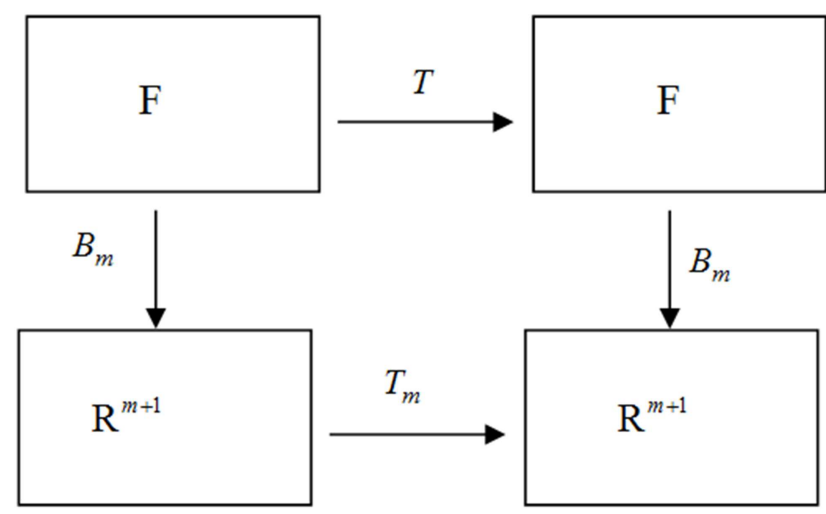

Fig. 1. Relation between $T$ and $T_{m}$.

Lemma 4. Let $\bar{x}_{i}-\bar{x}_{i-1}=\frac{\bar{x}_{m}-\bar{x}_{0}}{m}$, for $i=1,2, \ldots, m$. Let $T$ and $T_{m}$ be defined by the same $s_{i}, c_{i} d_{i}, i=1,2, \ldots, n$. Then for $g \in \mathrm{F}$, we have

$$
\left\|T_{m} B_{m} g-B_{m} T g\right\|_{X_{m}} \rightarrow 0
$$

Proof. By the definitions of $B_{m}$ and $T_{m}$, we get

$$
\begin{gathered}
B_{m} g=\left(g\left(\bar{x}_{0}\right), g\left(\bar{x}_{1}\right), \ldots, g\left(\bar{x}_{m}\right)\right), T_{m} B_{m} g=\left(\bar{g}_{0}, \bar{g}_{1}, \ldots, \bar{g}_{m}\right)( \\
\bar{g}_{i}=s_{l}\left(g_{k}+g_{k+1}\right) / 2+p_{l}\left(u_{l}^{-1}\left(\bar{x}_{i}\right)\right) \\
\bar{x}_{i} \in I_{l}, \quad \bar{x}_{k} \leq u_{l}^{-1}\left(\bar{x}_{i}\right) \leq \bar{x}_{k+1}
\end{gathered}
$$

and

$$
\begin{gathered}
\operatorname{Tg}(x)=s_{l} g\left(u_{l}^{-1}(x)\right)+p_{l}\left(u_{l}^{-1}(x)\right), x \in I_{l}, \\
B_{m} \operatorname{Tg}=\left(\operatorname{Tg}\left(\bar{x}_{0}\right), \operatorname{Tg}\left(\bar{x}_{1}\right), \ldots, \operatorname{Tg}\left(\bar{x}_{m}\right)\right) .
\end{gathered}
$$

Therefore, we have

$$
\begin{aligned}
& \mid\left(T_{m} B_{m} g\right)_{i}-\left(B_{m} T g\right)_{i}|=| s_{l}\left(g_{k}+g_{k+1}\right) / 2+p_{l}\left(u_{l}^{-1}\left(\bar{x}_{i}\right)\right) \\
&-s_{l} g\left(u_{l}^{-1}\left(\bar{x}_{i}\right)\right)-p_{l}\left(u_{l}^{-1}\left(\bar{x}_{i}\right)\right) \mid= \\
&=\left|s_{l}\left(\left(g_{k}+g_{k+1}\right) / 2-g\left(u_{l}^{-1}\left(\bar{x}_{i}\right)\right)\right)\right| \rightarrow 0 \quad(m \rightarrow \infty),
\end{aligned}
$$

which gives(40).

If $T$ and $T_{m}$ are defined by the same $s_{i}, c_{i}$ and $d_{i}$, $i=1,2, \ldots, n$, then since contraction constants of $T$ and $T_{m}$ are given by $s_{i}, i=1,2, \ldots, n$, the elements of $\mathcal{T}$ and $T_{m}$ have the same contraction constant $c$.

Theorem 3. Let $T$ and $T_{m}$ be defined by the same $s_{i}, c_{i}$ and $d_{i}, i=1,2, \ldots, n$. If for $\bar{x}_{0}, \bar{x}_{1}, \ldots, \bar{x}_{m}, u_{l}^{-1}\left(\bar{x}_{i}\right) \in$ $\left\{\bar{x}_{0}, \bar{x}_{1}, \ldots, \bar{x}_{m}\right\}, \quad \bar{x}_{i} \in I_{l}$ and $l \in\{1, \cdots, n\}$, then we get $B_{m} f_{T}=T_{m} B_{m} f_{T}$ and

$$
\left\|B_{m} f_{T}-\hat{z}\right\| \leq \frac{1}{1-c}\left\|T_{m} \hat{z}-\hat{z}\right\|
$$

Proof. Since $f_{T}$ is the fixed point of T, we have

$$
f_{T}(x)=T f_{T}(x)=s_{l} \cdot f_{T}\left(u_{l}^{-1}(x)\right)+p_{l}\left(u_{l}^{-1}(x)\right), \quad x \in I_{l},
$$

and by the definitions of $B_{m}$ and $T_{m}$, we get

$$
\left(B_{m} f_{T}(x)\right)_{i}=s_{l} \cdot f_{T}\left(u_{l}^{-1}\left(\bar{x}_{i}\right)\right)+p_{l}\left(u_{l}^{-1}\left(\bar{x}_{i}\right)\right) .
$$

Since

$$
B_{m} f_{T}=\left(f_{T}\left(\bar{x}_{0}\right), \ldots, f\left(\bar{x}_{m}\right)\right),
$$

we have

$$
T_{m} B_{m} f_{T}=T_{m}\left(f_{T}\left(\bar{x}_{0}\right), \ldots, f\left(\bar{x}_{m}\right)\right)
$$

and

$$
\left(T_{m} B_{m} f_{T}\right)_{i}=s_{l} \cdot f_{T}\left(u_{l}^{-1}\left(\bar{x}_{i}\right)\right)+p_{l}\left(u_{l}^{-1}\left(\bar{x}_{i}\right)\right) .
$$

Hence, we have $B_{m} f_{T}=T_{m} B_{m} f_{T}$ and

$$
\begin{aligned}
& \left\|\mathrm{B}_{\mathrm{m}} \mathrm{f}_{\mathrm{T}}-\hat{\mathrm{z}}\right\| \leq\left\|\mathrm{B}_{\mathrm{m}} \mathrm{f}_{\mathrm{T}}-\mathrm{T}_{\mathrm{m}} \hat{\mathrm{z}}\right\|+\left\|\mathrm{T}_{\mathrm{m}} \hat{\mathrm{z}}-\hat{\mathrm{z}}\right\| \\
& =\left\|\mathrm{T}_{\mathrm{m}} \mathrm{B}_{\mathrm{m}} \mathrm{f}_{\mathrm{T}}-\mathrm{T}_{\mathrm{m}} \hat{\mathrm{z}}\right\|+\left\|\mathrm{T}_{\mathrm{m}} \hat{\mathrm{z}}-\hat{\mathrm{z}}\right\| \\
& \leq c\left\|B_{m} f_{T}-\hat{z}\right\|+\left\|T_{m} \hat{z}-\hat{z}\right\|
\end{aligned}
$$

Thus $\left\|B_{m} f_{T}-\hat{z}\right\| \leq \frac{1}{1-c}\left\|T_{m} \hat{z}-\hat{z}\right\|$.

Lemma 5. [1] Let $\mathrm{X}$ be aBanach space and $\mathrm{T}$ a contraction operator on $\mathrm{X}$ with the contraction constant c. Let $f_{T}$ be the fixed point of T. If for $f \in X,\|f-T f\|<\varepsilon$, then

$$
\left\|f_{T}-f\right\|<\varepsilon /(1-c) .
$$

Denote $\varepsilon_{1}=\left\|T_{m}^{*} \hat{z}-\hat{z}\right\|$ and 


$$
\varepsilon_{2}=\left\|T_{m}^{*} B_{m} f_{T^{*}}-B_{m} f_{T_{m}^{*}}\right\|=\left\|T_{m}^{*} B_{m} f_{T^{*}}-B_{m} T^{*} f_{T_{m}^{*}}\right\| .
$$

Theorem 4. Let $f_{T^{*}}$ be the fixed point of $T^{*}$ defined by the solution $\left(y_{0}^{*}, \ldots, y_{n}^{*}\right)$ of (37). Then we have

$$
\left\|B_{m} f_{T^{*}}-\hat{z}\right\| \leq\left(\varepsilon_{1}+\varepsilon_{2}\right) /(1-c),
$$

where $c$ is the contraction constant of the contraction operator $T_{m}^{*}$. Especially, $\varepsilon_{2}=0$ under the conditions of Theorem 3.

Proof. We caneasily see that

$$
\left\|B_{m} f_{T^{*}}-\hat{z}\right\| \leq\left\|B_{m} f_{T^{*}}-f_{T_{m}^{*}}\right\|+\left\|f_{T_{m}^{*}}-\hat{z}\right\| .
$$

From Lemma 4, we have $\left\|T_{m}^{*} B_{m} f_{T^{*}}-B_{m} f_{T^{*}}\right\| \rightarrow 0$.

Let us denote $p:=T_{m}^{*} B_{m} f_{T^{*}}-B_{m} f_{T^{*}}, p=\left(p_{1}, \ldots, p_{m}\right)$, For $\bar{x}_{i} \in I_{l}$, there exists a $k \in\{0,1, \cdots, m\}$ such that $\bar{x}_{k} \leq u_{l}^{-1}\left(\bar{x}_{i}\right) \leq \bar{x}_{k+1}$. Then we get

$$
\begin{aligned}
p_{i} & =s_{l}\left(f_{T^{*}}\left(\bar{x}_{k}\right)+f_{T^{*}}\left(\bar{x}_{k+1}\right)\right) / 2+p_{l}\left(u_{l}^{-1}\left(\bar{x}_{i}\right)\right) \\
& -s_{l} f_{T^{*}}\left(u_{l}^{-1}\left(\bar{x}_{i}\right)\right)-p_{l}\left(u_{l}^{-1}\left(\bar{x}_{i}\right)\right) \\
& =s_{l}\left(\left(f_{T^{*}}\left(\bar{x}_{k}\right)+f_{T^{*}}\left(\bar{x}_{k+1}\right)\right) / 2-f_{T^{*}}\left(u_{l}^{-1}\left(\bar{x}_{i}\right)\right)\right) .
\end{aligned}
$$

From Lemma 5, we have

$$
\left\|B_{m} f_{T^{*}}-f_{T_{m}^{*}}\right\| \leq \varepsilon_{2} /(1-c),\left\|f_{T_{m}^{*}}-\hat{z}\right\| \leq \varepsilon_{1} /(1-c),
$$

where $c=\max \left\{\left|s_{i}\right|, \quad i=1, \ldots, n\right\}$.

By (51) and(53),we get(50).

\section{Examples of Calculation}

Example 1. Let $P$ be a data set given by $P=\left\{\left(\bar{x}_{i}, z_{i}\right) \in R^{2}: i=0,1, \ldots, 10\right\}=\{(0,3.6),(0.1,5.1)$, $(0.2,5.6),(0.3,6.3),(0.4,6.0),(0.5,5.4),(0.6,5.6),(0.7,5.0)$, $(0.8,4.2),(0.9,3.2),(1,1.7)\}$.

Let $\left\{x_{0}, x_{1}, x_{2}, x_{3}, x_{4}\right\}=\{0,0.2,0.5,0.7,1\}$,

$$
\Delta=\left\{\left(0, y_{0}\right),\left(0.2, y_{1}\right),\left(0.5, y_{2}\right),\left(0.7, y_{3}\right),\left(1, y_{4}\right)\right\}
$$

$$
S=\left\{s_{1}, s_{2}, s_{3}, s_{4}\right\}=\left\{\frac{1}{3}, \frac{2}{5}, \frac{1}{6}, \frac{1}{7}\right\} .
$$

Then, $I_{1}=[0,0.2], I_{2}=[0.2,0.5], I_{3}=[0.5,0.7]$ and $I_{4}=[0.7,1]$.

Let $J_{1}=[0,1], J_{2}=[0,1], J_{3}=[0,1], J_{4}=[0,1]$.

By (1) and (3), we have $u_{1}(x)=0.2 x, u_{2}(x)=0.3 x+0.2$, $u_{3}(x)=0.2 x+0.5, \quad u_{4}(x)=0.3 x+0.7$ and by (15) and $(16)$, $c_{i}, d_{i}, i=1, \cdots, 4$ are given by

$$
\mathrm{c}=\left\{\begin{array}{c}
-0.666667 y_{0}+y_{1}-0.333333 y_{4}, \\
0.4 y_{0}-y_{1}+y_{2}-0.4 y_{4},
\end{array}\right.
$$

$$
\begin{gathered}
0.166667 y_{0}-y_{2}+y_{3}-0.166667 y_{4}, \\
\left.0.142857 y_{0}-y_{3}+0.857143 y_{4}\right\}
\end{gathered}
$$

$\mathrm{d}=\left\{0.666667 y_{0},-0.4 y_{0}+y_{1},-0.166667 y_{0}+y_{2},-0.142857\right.$

$$
\left.y_{0}+y_{3}\right\} \text {. }
$$

Then, we have $\left(y_{0}, y_{1}, y_{2} y_{3}, y_{4}\right)=(3.16738,4.97274$, $5.05272,4.84987,1.66452)$ from the problem (37).

Hence, we getc $=(2.30631,0.681127,0.0476312,-2.97066)$, $\mathrm{d}=(2.11159,3.70579,4.52482,4.39739)$.

The attractor of IFS $\left\{\mathrm{R}^{2}: w_{1}, w_{2}, w_{3}, w_{4}\right\}$, $w_{i}\left(\begin{array}{l}x \\ y\end{array}\right)=\left(\begin{array}{c}u_{i}(x) \\ s_{i} y+c_{i} x+d_{i}\end{array}\right), i=1,2,3,4$, is the graph of the found least squares fractal approximation(see Fig 2).

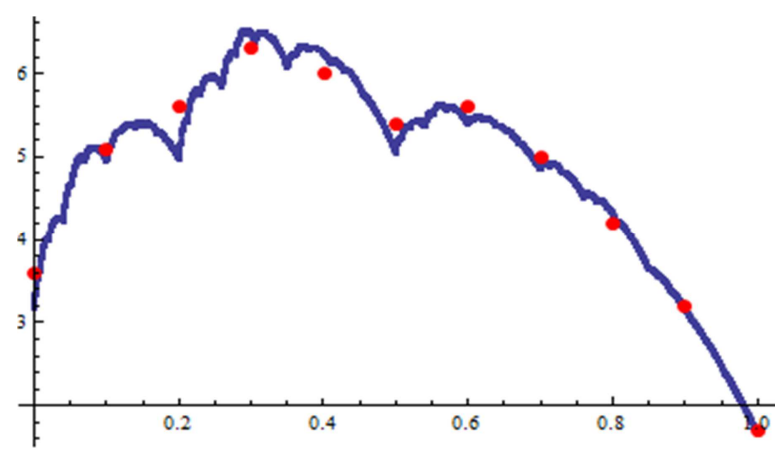

Fig. 2. LSFA of a data set. The points are one of the data set.

Example 2. Calculate LSFA of a coastline with a data set taken from the coastline in Fig 3.

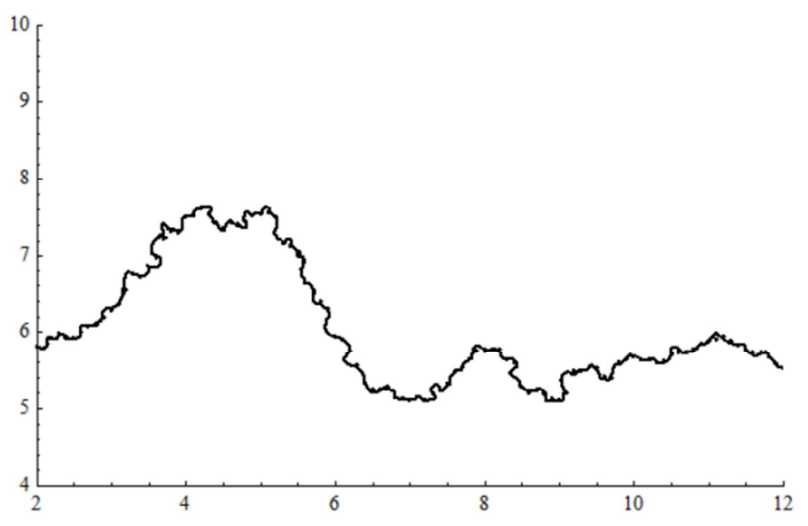

Fig. 3. The coastline.

We take the following data set: $\mathrm{P}=\{(2.0,5.82),(2.1,5.86)$, (2.2, 5.9), (2.3, 5.92), (2.4, 5.92), (2.5, 5.92), (2.6, 6.0), (2.7, 6.22), (2.8, 6.29), (2.9, 6.31), (3.0, 6.29), (3.1, 6.27), (3.2, $6.39),(3.3,6.63),(3.4,7.15),(3.5,7.29),(3.6,7.47),(3.7$, 
7.49), (3.8, 7.55), (3.9, 7.59), (4.0, 7.61), (4.1, 7.54), (4.2, 7.42), (4.3, 7.26), (4.4, 7.28), (4.5, 7.36), (4.6, 7.36), (4.7, $7.40),(4.8,7.40),(4.9,7.36),(5.0,7.32),(5.1,7.42),(5.2$, $7.38),(5.3,7.38),(5.4,7.28),(5.5,7.01),(5.6,6.75),(5.7$, $6.54),(5.8,6.23),(5.9,5.52),(6.0,5.48),(6.1,5.44),(6.2$, $5.36),(6.3,5.46),(6.4,5.34),(6.5,5.18),(6.6,5.24),(6.7,5.28)$, $(6.8,5.16),(6.9,5.14),(7.0,5.12),(7.1,5.16),(7.2,5.12\},(7.3$, 5.10), (7.4, 5.06), (7.5, 5.1), (7.6, 5.48), (7.7, 5.82), (7.8, 5.98), $(7.9,5.98),(8.0,5.84),(8.1,5.70),(8.2,5.62),(8.3,5.58),(8.4$, $5.4),(8.5,5.28),(8.6,5.20),(8.7,5.24),(8.8,5.2),(8.9,5.14)$, $(9.0,5.12),(9.1,5.20),(9.2,5.50),(9.3,5.50),(9.4,5.54),(9.5$, 5.56),(9.6, 5.42), (9.7, 5.40), (9.8, 5.6), (9.9, 5.64), (10.0, 5.68), (10.1, 5.64), (10.2, 5.64), (10.3, 5.60), (10.4, 5.62), (10.5, 5.70), (10.6, 5.74), (10.7, 5.74), (10.8, 5.78),(10.9, 5.86), (11.0, $5.88),(11.1,5.88),(11.2,5.70),(11.3,5.76),(11.4,5.84),(11.5$, $5.78),(11.6,5.74),(11.7,5.74),(11.8,5.68),(11.9,5.58)$, $(12.0,5.52)\}$

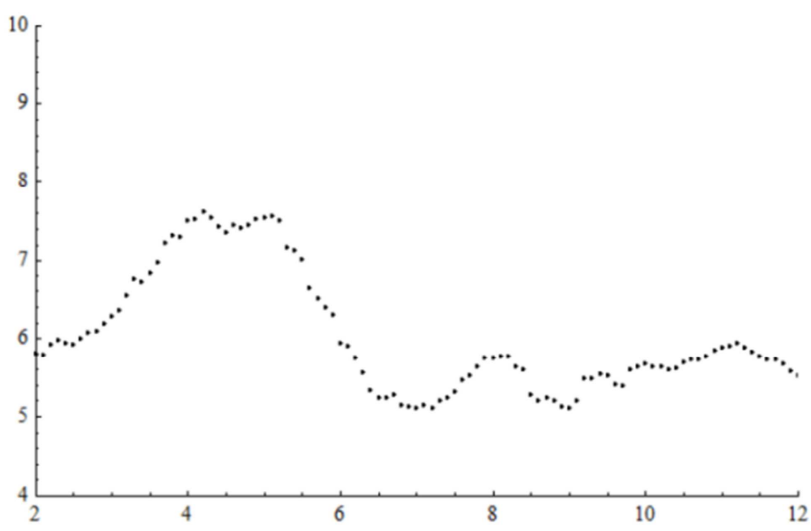

Fig. 4. A data set of the coastline.

Then, we have $I=[2, \quad 12]$, $\Delta=\left\{x_{0}, x_{1}, x_{2}, x_{3}, x_{4}, x_{5}, x_{6}, x_{7}, x_{8}, x_{9}, x_{10}\right\}, I_{1}=[2,3]$, $I_{2}=[3,4], I_{3}=[4,5], I_{4}=[5,6], I_{5}=[6,7], I_{6}=[7,8]$, $I_{7}=[8,9], \quad I_{8}=[9,10], \quad I_{9}=[10,11], \quad I_{10}=[11,12]$, $\left(y_{0}, y_{1}, y_{2}, y_{3}, y_{4}, y_{5}, y_{6}, y_{7}, y_{8}, y_{9}, y_{10}\right)=(5.71008$, $6.22253,7.4376, \quad 7.58819, \quad 5.95495, \quad 4.91328, \quad 5.74059$, $5.12894,5.59619,5.83781,5.6046), \mathrm{c}=(0.0522994,0.122562$, $0.0161142,-0.162269,-0.103113,0.0837858,-0.0601096$, $0.0477794,0.0252167,-0.0222661), \mathrm{d}=(5.03448,5.4064$, 6.83436, 7.34172, 5.59017, 4.1747, 5.2898, 4.46238, 4.97475, 5.31133).

The attractor of IFS $\left\{\mathrm{R}^{2}: w_{1}, w_{2}, \cdots, w_{10}\right\}$, $w_{i}\left(\begin{array}{l}x \\ y\end{array}\right)=\left(\begin{array}{c}u_{i}(x) \\ s_{i} y+c_{i} x+d_{i}\end{array}\right), i=1,2, \cdots, 10$ is the graph of the found least squares fractal approximation (see Fig 5).

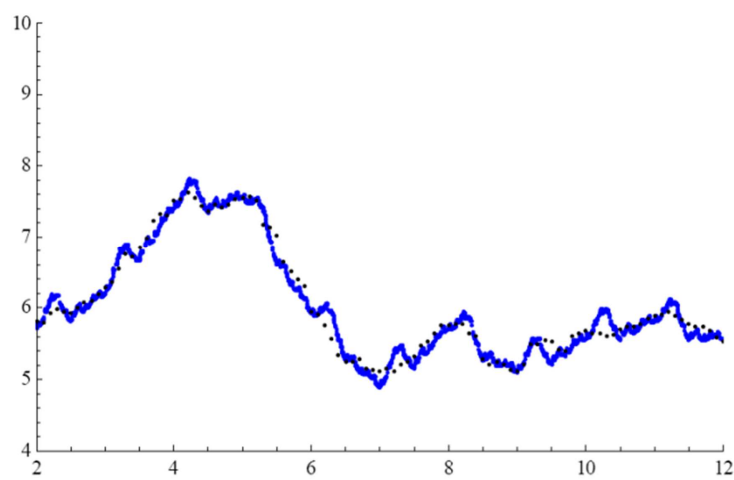

Fig. 5. The fractal approximation of the coastline.

\section{References}

[1] M. F. Barnsley, Fractals Everywhere, Academic Press, New York, 1988.

[2] M. F. Barnsley, Fractal functions and interpolation, Constr. Approx. 2(1) (1986) 303-329.

[3] M. F. Barnsley, J. H. Elton, D. P. Hardin, Recurrent iterated function systems, Constr. Approx. 5(1) (1989) 3-31.

[4] P. Bouboulis, L. Dalla, A general construction of fractal interpolation functions ongridsof $R^{n}$, European J. Appl. Math. 18(4) (2007) 449-476.

[5] P. Bouboulis, L. Dalla, Fractal interpolation surfaces derived from fractal interpolationfunctions, J. Math. Anal. Appl. 336(2) (2007) 919-936.

[6] P. Bouboulis, L. Dalla, V. Drakopoulos Construction of recurrent bivariate fractal interpolation surfaces and computation of their boxcounting dimension, J. Approx. Theory 141 (2006) 99-117.

[7] P. Bouboulis, M. Mavroforakis, Reproducing kernel Hilbert spaces and fractal interpolation, J. Comput. Appl. Math. 235 (2011) 3425-3434.

[8] A. K. B. Chand, G. P. Kapoor, Generalized cubic spline fractal interpolation functions, SIAM J. Numer. Anal. 44(2) (2006) 655-676.

[9] L. Dalla, Bivariated fractal interpolation functions on grids, Fractals 10(1) (2002) 53-58.

[10] Z. G. Feng, Y. Z. Feng, Z. Y. Yuan, Fractal interpolation surfaces with function verticalscaling factors, Appl. Math. Lett. 25(11) (2012) 1896-1900.

[11] R. Malysz, The Minkowski dimension of the bivariate fractal interpolation surfaces, Chaos Solitons Fractals 27(5) (2006) $1147-1156$.

[12] P. R. Massopust, Fractal Functions and their applications, Chaos Solitons Fractals 8(2) (1997) 171-190.

[13] W. Metzler, C. H. Yun, Construction of fractal interpolation surfaces on rectangulargrids, Internat. J. Bifur. Chaos 20(12) (2010) 4079-4086.

[14] M. A. Navascues, M. V. Sebastian, Generalization of Hermite functions by fractalinterpolation, J. Approx. Theory 131(1) (2004) 19-29. 
[15] S. Lonardi, P. Sommaruga, Fractal image approximation and orthogonal bases, SignalProcess. Image Commun. 14(5) (1999) 413-423.

[16] Y. S. Kang, C. H. Yun, A construction of best fractal approximation, Electron. J. Math. Anal. Appl. 2(2) (2014) 144-151.
[17] C. H. Yun, H. C. Choi, H. C. O, Construction of fractal surfaces by recurrent fractal interpolation curves, Chaos Solitons Fractals 66(2014) 136-143.

[18] H. Zhang, R. Tao, S. Zhou, Y. Wang, Wavelet-based fractal function approxmation,J. Syst. Engrg. Electron. 10(4) (1999) 60-66. 